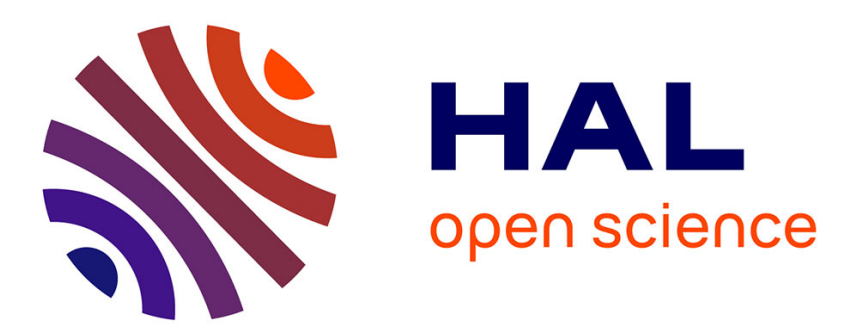

\title{
Rapid morphological exploration with the Poppy humanoid platform.
}

Matthieu Lapeyre, Steve N'Guyen, Alexandre Le Falher, Pierre-Yves Oudeyer

\section{To cite this version:}

Matthieu Lapeyre, Steve N'Guyen, Alexandre Le Falher, Pierre-Yves Oudeyer. Rapid morphological exploration with the Poppy humanoid platform.. 2014 IEEE-RAS International Conference on Humanoid Robots, Nov 2014, Madrid, Spain. pp.8. hal-01096344

HAL Id: hal-01096344

https://hal.inria.fr/hal-01096344

Submitted on 17 Dec 2014

HAL is a multi-disciplinary open access archive for the deposit and dissemination of scientific research documents, whether they are published or not. The documents may come from teaching and research institutions in France or abroad, or from public or private research centers.
L'archive ouverte pluridisciplinaire HAL, est destinée au dépôt et à la diffusion de documents scientifiques de niveau recherche, publiés ou non, émanant des établissements d'enseignement et de recherche français ou étrangers, des laboratoires publics ou privés. 


\title{
Rapid morphological exploration with the Poppy humanoid platform.
}

\author{
Matthieu Lapeyre ${ }^{1}$, Steve N'Guyen ${ }^{1}$, Alexandre Le Falher ${ }^{1}$ and Pierre-Yves Oudeyer ${ }^{1}$
}

\begin{abstract}
In this paper we discuss the motivation and challenges raised by the desire to consider the morphology as an experimental variable on real robotic platforms as well as allowing reproducibility and diffusion in the scientific community. In this context, we present an alternative design and production methodology that we have applied to the conception of Poppy, the first complete 3D printed open-source and openhardware humanoid robot. Robust and accessible, it allows exploring quickly and easily the fabrication, the programming and the experimentation of various robotic morphologies. Both hardware and software are open-source, and a web platform allows interdisciplinary contributions, sharing and collaborations. Finally we conduct an experiment to explore the impact of four different foot morphologies on the robot's dynamic when it makes a footstep. We show that such experimentation can easily be achieved and shared in couple of days at almost no cost.
\end{abstract}

\section{INTRODUCTION}

First robots behaviors (e.g. the Grey Walter's turtles) were dependent on their bodies and in particular, controlled using pre-wired electronic circuits [1]. With the introduction of numerical computers in the robotics field, researchers saw an opportunity to create behaviors and artificial intelligence no more dependent on the actual robot body. Eventually, researchers no longer saw the physical incarnation as an essential component of their research and were convinced that using the notion of computation or abstract symbol manipulation, it would be possible to reproduce interesting abilities similar to human ones [2] [3]. With such paradigm, the "intelligence" or the ability for an agent to achieve a task is determined by its capacity to compute complex internal models, the body is reduced to a noisy interface between the abstract algorithm and the real world.

In the late 80's emerges a novel paradigm thanks to researchers such as Rodney Brooks [4], Luc Steels [5] or Rolf Pfeifer [6]. The embodied artificial intelligence rejects the symbolic approach and postulates that it is not possible to have intelligence without an actual robot body associated with its ecological niche [4]. Following this paradigm, several researchers tried to tackle challenges in which the classical cognitivist approach failed e.g. the understanding of natural forms of intelligence that require a direct interaction with the real world [7].

Thus an interesting evolution of the last decades is the demonstration of the importance of the morphology for sensorimotor control, cognition and development [8] [9] [10].

\footnotetext{
*This research was partially funded by ERC Starting Grant EXPLORER 240007.

1 INRIA Flowers Team, Bordeaux, France matthieu.lapeyre, steve.nguyen, pierre-yves.oudeyer at inria.fr
}

As Rodney Brooks argued, the world is its own best model [4] and simulators cannot realistically handle the complexity of the real physic with multi-point contacts, soft materials compliance and frictions or unpredicted multimodal interactions. Therefore, considering the robot morphology defined as any characteristic which defines the physical structure of the robot such as link sizes, number of links, joint characteristics, mass distribution, actuator characteristics, material properties, sensor characteristics and sensor placements [11], we should not only take care of the robot body design but introduce the morphology as an experimental variable and conduct experiments in the real world [12].

Thus it is necessary to build robotic platform allowing to experiment in the real world. Among all kind of robots, research in humanoid robotics has been thriving in the recent years [13] [14], both due to their predicted relevance for personal and assistive robotics [15], and because of the scientific challenges raised by robotics with regards to cognition [16], natural communication [17], biped locomotion [18] and fullbody physical interaction with the environment [19].

In addition, humanoid robots can be great tools to study human being and eventually contribute to a better understanding of Human's behaviors and abilities [20] [21].

However conducting such experiments in the real world is challenging: considering the morphology as an experimental variable raised two major problems:

- how can we obtain an experimental robotic platform with both a morphology that can be changed easily and quickly and the capacity to act robustly in the real world?

- how can we make sure that this platform, particularly the hardware, can be diffused and reused in the research community?

Unfortunately current robotic platforms are not suitable to address such challenges.

On one hand, commercial robots such as Nao [22], Darwin Op [23], Nimbro Op [24] or iCub [25] are easily accessible and easy to use. Yet they provide a "traditional" morphology (e.g. limited compliance, rigid torso, big feet, powerful actuation) and they do not permit the modification of their morphology. In most case, they are not open source and/or the hardware is to complicated/expensive to be modified.

On the other hand, lab prototypes are mainly handcrafted and specifically tuned which make them almost impossible to be reproduced in another lab.

The main issue of these robots are the chosen approaches and technologies used to design and produce them. Indeed, the classic way to design and produce robot is a complicated, 
time-consuming and an expensive process involving specific upfront tooling and complex manufacturing processes.

Similarly to the Locomorph project [26] which offer a multi-purpose hardware kit allowing to quickly create robot and study the impact of several morphological properties such as link length, joint stiffness or mass distribution, we explored how we could build novel kind of robotic platform allowing to quickly tune morphological parameters.

Within this context we built a whole new humanoid robot called Poppy (see Fig. 1) presented in details in [27]. This humanoid robot was designed to easily and quickly conduct scientific experiments on sensorimotor learning, exploring morphological properties, and human-robot interaction. As an experimental robotic platform, Poppy was designed to be affordable, lightweight, robust and safe, easy to use, highly-hackable and fast and easy to duplicate or modify with the goal to be easily reproducible and used by other lab thanks to an open source distribution (hardware and software). This was achieved thanks to $3 \mathrm{D}$ printing techniques, affordable off-the-shell components and optimized modular design.

In the next section, we will present the challenges we have addressed. Then in section III. we will present the Poppy platform and its associated methodology and design processes made to tackle these challenges. Finally we will conduct an experimentation as illustration of the use of Poppy for exploring morphological variants (here a comparison of several foot conception) and the sharing results with the scientific community in section IV

\section{Challenges}

As we discussed in the introduction, the role of morphology appears as a fascinating open field of research but current robotic platforms - both prototype and commercial platform - do not permit to really consider their morphology as an experimental variable or are not reproducible.

In this context, creating a platform reproducible everywhere without special tooling or skills, and in which the morphology can be freely explored raises methodological and design process challenges:

\section{A. Make the morphology variable}

Current robotic platforms, in particular humanoid ones, have mechanical parts either handcrafted or produced using classic machining technique based on milling or casting various metal alloys or plastic. These techniques require specific upfront tooling which make the production of small batch really expensive. In this context, current robotic platform cannot have their morphology modified because it would require to redo most of the production process. The same issues appear with electronics and the robot sensor space, which is, in most cases, frozen.

Therefore the classic way to design and produce robot is not adapted to the free exploration of the robot morphology, novel design and production methods have to be used.

\section{B. Create reproducible robot prototype}

Several interesting robotic platforms explore key aspects about the robot morphology, we can cite the complex bioinspired artificial muscles actuator network of Kenshiro [28] , or the impressive walking ability using semi-passive dynamics of Denise [29]. Unfortunately, none of these robots can be - and has ever been - transferred to another lab. Indeed their production requires specific tooling, tuning and handcrafting only few skilled people have.

To enhance scientific impact, our work should be reproducible in another lab. This is essential as it permits the validation of scientific results presented in publications, and it enables cumulative science which permits to accelerate the development of novel technologies. We are especially attentive to the following criteria:

- Precision, stationary Experiments should be reproducible, repeatable, implying that the robot morphology properties should be stationary.

- Easy and fast to duplicate: such a reuse of the robotic platform requires that it is easy and fast to duplicate in any laboratory and so, does not rely on specific tooling or exotic components.

- Affordable: to ensure a wide spread and scientific diffusion, a key aspect is to keep the cost of platform relatively low.

\section{Keep robotic platform simple and easy-to-use}

The robotics field is intrinsically multidisciplinary. A robot itself requires technologies coming from mechanics, electronics and computer sciences, but the scientific impact of robotics contributions can be wider and reach non-engineering fields such as human, social or biological sciences. Thus, robotics field is an expert field where nobody can be expert in each required skills. We have to take into account the fact that the end user may certainly be expert in one specific field but beginner in the other fields. This mean that in each field, the designed robot has to be simple enough to be understood and used by beginners as well as having enough potential to be pertinent for expert users.

In the next sections, we will suggest novel approaches and design processes to create and produce robotic platform allowing a free exploration of their control and morphology through experimentation in the real world while being easily reproducible in the research community.

\section{THE POPPY PROJECT}

Most researchers can attest to the difficulty and frustration faced while conducting robotic experimentation in the real world. We are challenged daily by bugs, technical issues, unpredicted events and side effects. While a bug in software can be fixed, an error with a hardware platform can cause damage to the robot and postpone the results of an experiment by several weeks.

Therefore many researchers in robotics avoid technical issues associated with the real world experimentation by using 


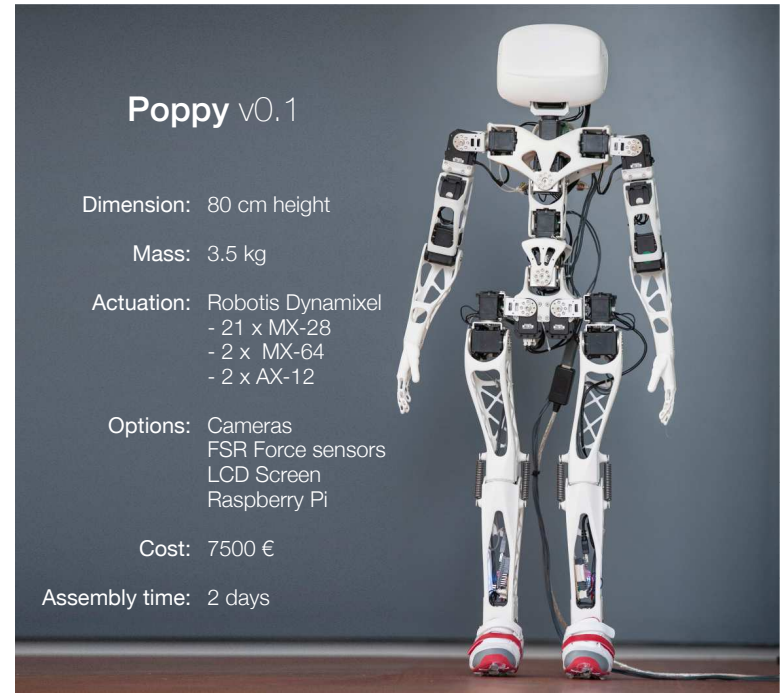

Fig. 1: The Poppy v0.1.5 platform specs overview.

simple models and physical simulation [30]. An efficient experimental platform should not break itself while acting in the real world or at least, it should be easy to repair it in case of problem. Moreover, considering morphology as an experimental variable raised major challenges: how can we easily change it on a real robotic platform and share it with the scientific community (see section III) ?

Throughout our work on building cognitive and developmental learning algorithms ([31],[32]), we have experienced these issues, especially while building and using Acroban [?]. Much time has been spent debugging non-robust technologies but it has been very instructive for understanding those that are efficient and those that should be avoided. Therefore Poppy has been designed based on the background experience we have acquired building using robots acting in the real world.

- Robustness and Safety: Demanding and lengthy realworld experimentation necessitates that the robot be robust and safe. It should be able to sustain experiments and fall down without easily breaking. At the same time, one should ensure that physical interaction with the robot is safe for humans.

- Precision, stationary: Experiments should be repeatable, implying that the robot properties should be stationary.

- Breakable, repairable: Breaking should not be costly and the robot should be easily repairable.

- Transportable: To allow for experiments in natural environments, possibly involving interaction with nontechnical humans, the robot should be transportable outside the laboratory.

- Easy and fast to duplicate: If the robotic platform is to be reused in this way, it must be easy and fast to duplicate.

- Affordable: To ensure widespread use, a key factor is to keep the cost of the platform relatively low. If more labs can be involved, the scientific impact can be greater.
We will present here the chosen approach for its conception allowing both freely exploring morphological variants and diffusing results in the research community. Then we will provide an overview of the platform and how we can actually use it to conduct such experiments.

\section{A. Design methodology}

We suggest exploring an alternative design methodology driven by the desire to:

- freely explore morphological properties,

- reduce the amount of time required between an idea and its experimentation on an actual robotic platform in the real life,

- keep experimental work reproducible in other labs,

- provide robust, easy to use, highly hackable and open source hardware and software modules.

We therefore chose an approach relied on the use of 3D printing for mechanical part, Arduino for the sensor acquisition and Python API for the control.

1) $3 D$ print mechanical parts: On one hand, we could produce classical manufactured mechanical parts but reconfigurable and adjustable, allowing for example to explore different length of a link or different center of mass position. However, this limits the morphological exploration to few dimensions with limited range.

On the other hand, since few years novel techniques, especially $3 \mathrm{D}$ printing, are revolutionizing the way we can produce objects. 3D printers open new horizons as they are able to produce parts which were, until now, either not possible or extremely expensive to produce using classical techniques while adding several key abilities:

- Accessible: 3D printed part can be obtained everywhere, either by personal printing or by using web service! 1

- Low cost: from few dozens of cents if produced on personal printer to few dozens of euros if outsourced through web services. Also the cost is not proportional to the part complexity, meaning designers are free to explore the shape they want with almost no constraints.

- Fast: The production took only few hours from scratch and does not require any specific upfront tooling.

- Skill-free: while the production process is fully numerical, few or no special skills are required.

- Multi-material, precise and robust: the current 3D printers can create precise (up to $0.1 \mathrm{~mm}$ ) part in different materials such as Polyamide, PLA, ABS and even titanium or flexible material. The obtained parts are robust and can often be used as final parts for several years.

- Reduces the number of part: 3D printing permits to print complex part and even assembled part as complex as bearing or gearbox. This means we can replace multiple parts that have to be assembled by a unique one ready-to-use right after its production.

${ }^{1}$ examples: i.materialise, shapeways or sculpteo 
These properties of the 3D printing process enable for the first time the exploration of morphological variant for mechanical parts. Indeed, it is now fast and low cost to create alternative design. Associated with modular architecture, we can easily and quickly change robot parts and conduct experiments. Also this process is compatible with the diffusion goals while it is simple and accessible anywhere with Internet connection and a mailing address.

2) Electronic architecture based on Arduino: Exploring the role of morphology does not only concern the mechanical properties but also the sensors apparatus i.e. which sensor is used and where it is placed on the body. While it is not yet possible to print complex electronic circuit, we preferred to rely on the Arduino hardware and software environment which make electronic board easily reconfigurable and compatible with a wide range of sensors. Also, low-level embedded programing skills are not necessary because the board micro-controller can be programmed using Arduino programming language which abstracts most of the complexity.

3) Easy to use python API: We designed a robust sensorymotor control API adapted to the hardware variability we have. We choose to use Python as it allows fast development, easy deployment on all operating system and quick scripting by non-necessary expert developers. It also offers a large variety of scientific and machine-learning libraries used in robotics (e.g. Numpy, Scipy, Scikit-learn).

4) Open source diffusion: As theses issues are encountered not only in our lab but also in the robotic community, and while the main aspect of such project is to create variability, reuses and modifications of initial design, we decided right from the beginning to make the platform easily accessible to anyone and we distribute it freely under open source licenses both for software and hardware.

\section{B. The Poppy platform}

Poppy is the first complete 3D printed open-source and open-hardware humanoid robot (see Fig. 11). Its 3D printed skeleton and its Arduino-based electronics are openhardware (Creative Commons). Its software is open-source (GPL V3), and allows programming beginners as well as advanced roboticists to control the robot in Python thanks to the PyPot library (www.poppy-project. org/pypot-library/). Its motors are common and widely used off-the-shell Robotis actuators (http://www . robotis.com/xe/dynamixel_en), and allow for compliant control and soft physical human-robot interaction. Poppy presents an original mechanical structure that permits to obtain a light structure with $3.5 \mathrm{~kg}$ for $84 \mathrm{~cm}$ height. Its current morphology takes insight from the human functional morphology: large number of articulation (25 motors), the limbs respect human proportions, it has five articulations in the trunk and its thigh is bended by a $6 \mathrm{deg}$ angle similar to the human which showed improvement on the biped stability [27].

Poppy is designed to conduct robotic experiments and integrates several key abilities in an easy-to-use robotic platform.

- Highly hackable: Poppy is fully modular (mechanic, electronic, software) meaning one can easily modify and adapted it to particular needs.

- Easy to duplicate: the overall time to assemble all mechanic components of Poppy takes about 2 days. Adding extra sensors is simplified by the use of Arduino electronic architecture.

- Robustness: Poppy is designed to be robust to falls and to allow long experimentation (e.g. several hours). Also, its conception, slightly under-actuated, prevents it from destructing itself if wrong moves occur.

- Easy to setup: we try to keep Poppy and modules as Plug'n'Play as possible.

- Affordable: to make Poppy widely accessible, we keep the cost relatively low. You can afford all components for 7500-8000€or thanks to its modularity, only use the parts of the robot needed.

Also we set up several web tools to support collaboration and sharing among members of the Poppy community: a portal web site (WWW . poppy-project . org), GitHub repositories for the hardware and software with associated wikis for documentation (www.github.com/poppy-project/), and a novel generation forum based on Discourse ${ }^{2}$ technology (forum.poppy-project.org).

\section{Exploring morphological variants with the Poppy plat- form}

Due to the need of exploring morphology alternatives, the use of $3 \mathrm{D}$ printing and rapid prototyping techniques is a central aspect of the project. These methods allow to really considering the morphology as an experimental variable. Indeed, thanks to the democratization of such technologies, exploring morphological variants is now both cheap and fast and thanks to a modular structure and the use of off-theshell components, we can reconfigure the robot in minutes and conduct experiment in the real world (see Fig. 2).

Also, because our work is fully open source and because we only use accessible components and production techniques, anyone can freely share and distribute (also commercially) upgrade, data or material associated with a use of Poppy.

In this paper, we suggest to illustrate the use of Poppy for exploring morphological variants with the example of foot design.

\section{EXPERIMENTS}

After discussing the design process and the motivation of creating a whole new humanoid platform in the previous section, we propose to conduct an experiment showing how the methodology used with Poppy can actually permit to easily and quickly explore the robot morphology in the real world.

We are currently working on a new design for Poppy's feet and exploring design similar to the "foot 1" (see table ??) so

www. discourse.org 


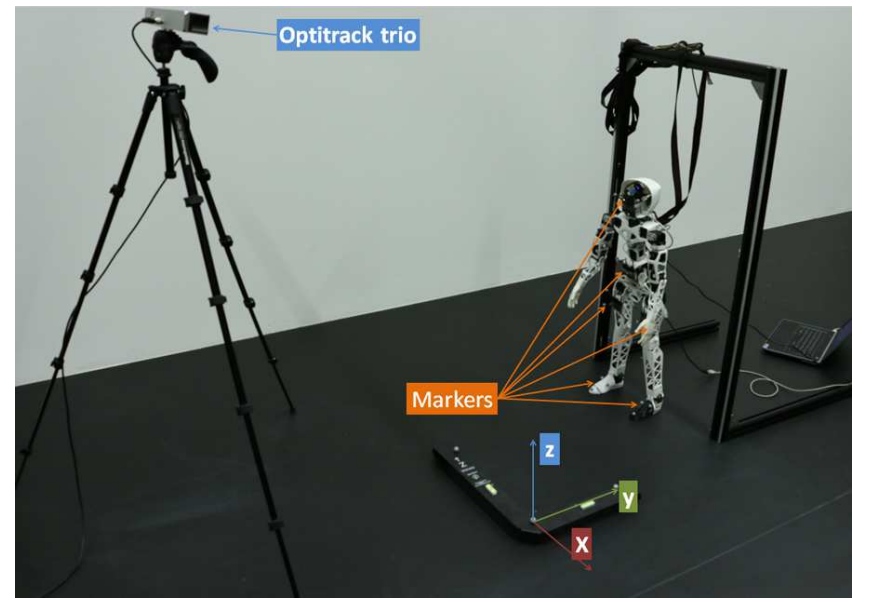

(a) Experimental setup. The robot is secured by slack strap on a gantry and tracked by an OptiTrack trio device. Markers are placed on the feet, hips, abdomen, and on the head
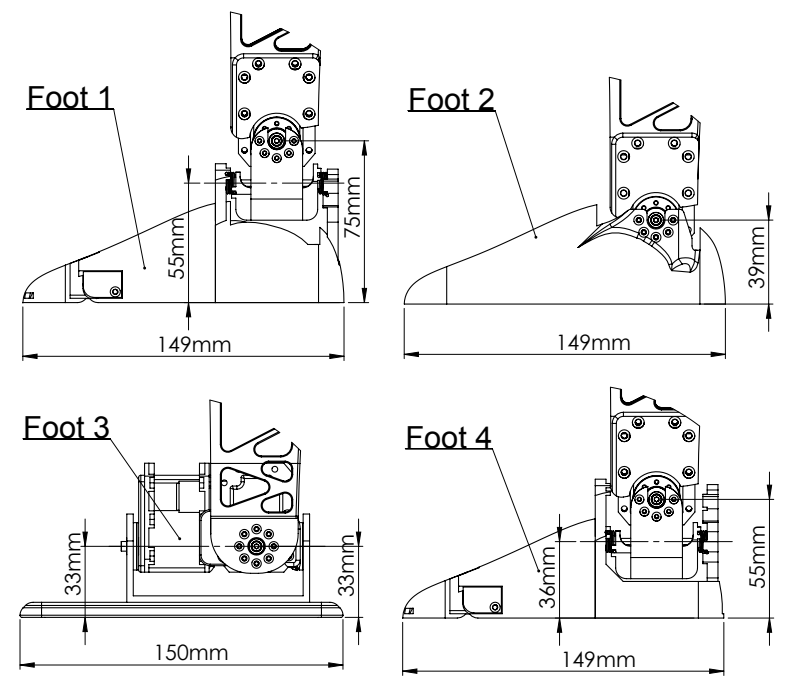

(b) Blueprints of the various foot designs studied in this experiments.

\begin{tabular}{l|cccc} 
Type & Foot 1 & Foot 2 & Foot 3 & Foot 4 \\
\hline $\begin{array}{l}\text { Double rotation } \\
\text { Toes }\end{array}$ & Passive & No & Active & Passive \\
& Yes & No & No & \\
& & & \\
\hline
\end{tabular}

(c) Table summarizing the different types of feet used.

Passive double-rotation: one active rotation (motor: Dynamixel MX 28) for the sagittal plan and a passive rotation for the frontal plan with two springs. Active double-rotation: A two motorized rotations (sagittal plan and frontal plan). No double-rotation: one motorized rotation (sagittal plan).

Toes: Indicates that the foot has toes.

Fig. 3: Descriptions of the four foot designs explored in this experiment.

we decided to use this context as illustration of the proposed method.

The aim of this experiment is to quickly explore the effect of feet morphology on stability. Here, we are particularly interested in the stability of the head after a stepping impact. These impacts are quite challenging to simulate realistically and the natural compliance of the Poppy platform makes it even more important to be tested on the real robot.

For the sake of lightness, the initial design of Poppy's feet only had one degree of freedom (pitch rotation). This configuration had the inconvenient of preventing a proper parallel foot/ground contact. Thus, we developed several different feet with two degrees of freedom. Along with a standard motorized 2 DoF flat foot design, we also wanted to explore passive joints with springs. The use of passive joints allows for both lightness and reactive torque for stability.

Moreover, it appeared that a proper foot/ground contact with a convenient friction was difficult to obtain based only on 3D printed material. One simple solution to this problem is to use a shoe which can provide a high friction and adapt slightly to the ground imperfections. Furthermore, this solution also allows keeping the feet close to humans ones. Thus, the tested feet (except the flat foot) were designed from a molding of the interior of a shoe. It is to be noted that we also included passive toes (with springs) on some of the tested feet for future work on locomotion. These toes should not have any significant impact on the tested criterion.

\section{A. Experimental setup}

For this experiment, the robot simply stands upright secured by a slack strap on a fixed gantry. Different markers on the robot are tracked by a motion capture system at $100 \mathrm{~Hz}$ (Natural Point OptiTrack). See figure 3a for more details.

Four different feet were tested (cf. Table ??). Three out of the four feet were tested both with and without shoes.

\section{B. Experiences}

The feet were tested with a very simple discrete movement, representative of the kind of impacts happening during walking. The robot performs a single step leftward with the left leg. The left foot is lifted $(3 \mathrm{~cm})$ and then put back on the ground with a slight lateral displacement towards the exterior 


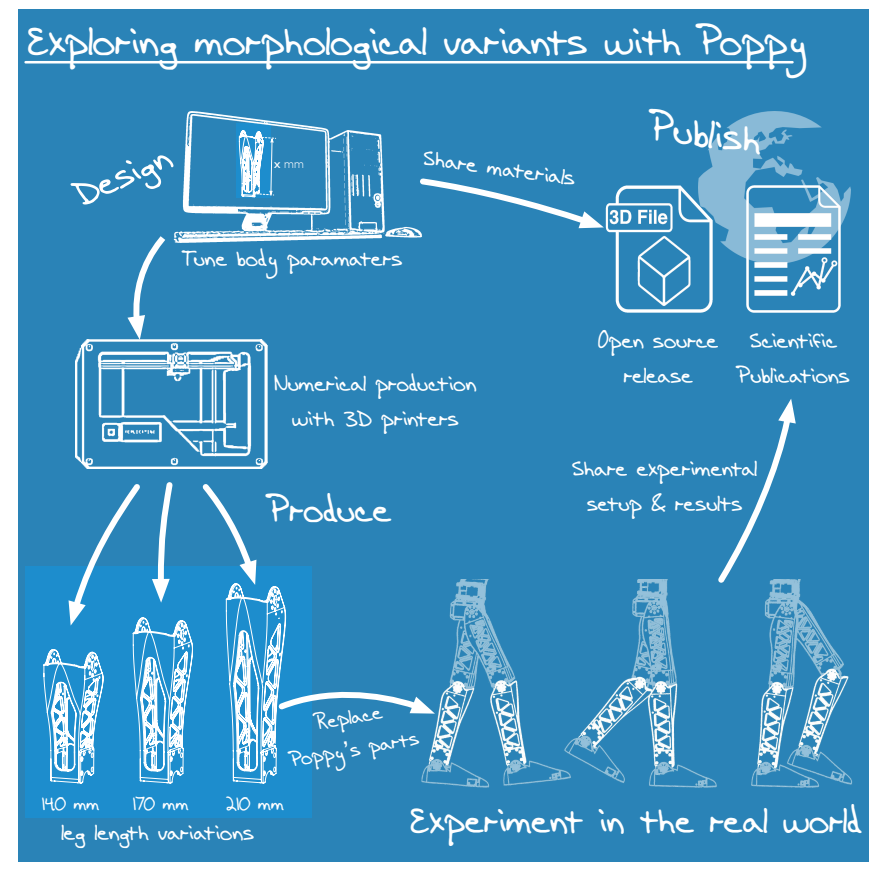

Fig. 2: Thanks to a modular design and the use of numerical 3D printing to produce mechanical part, the Poppy platform can be easily hacked to explore morphological variants. In just few days, we can conduct a whole set of experimentation. While the production technique used is low cost and easily reproducible, the material needed for a given experiment can be distribute along a scientific publication, preferentially under an open source license.

( $5^{\circ}$ at the level of the hip). The whole movement duration is about $0.4 \mathrm{~s}$ and repeated 20 times for each configuration.

\section{Results}

Figures 4,5 and 6 respectively show the evolution of the position of the head marker in the $x, y$ and $z$ axis for each tested foot. Dotted vertical lines indicate the beginning and the end of the leg movement.

These figures show that the dynamics of the robot is not trivial, even for the simple movement we tested, the standard deviation is not negligible and shows how chaotic the reaction of such impact can be. This particularity is another proof of the significance of the use of the experiment versus the simulation.

We can clearly see that the foot 3 (standard flat foot) behaves quite differently than other tested feet. In particular in the $x$ and $y$ directions, we see that with this foot the head tends to move more towards the exterior (left of the robot) and towards the rear.

Regarding the effect of the shoes, results are less clear but most of the time (except for the foot 1) differences occur between a given foot with and without shoe. The friction with the ground can explain these differences. Naked feet tends to slip more than with shoe.

This first experiment allowed us to determine that the use of an active double rotation of the ankle may not be

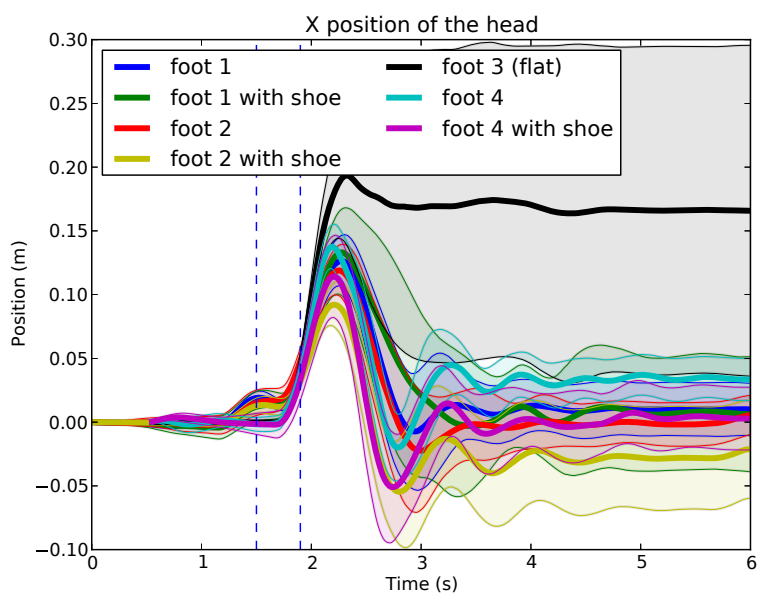

Fig. 4: Evolution of the position of the head in the $x$ axis for each tested foot.

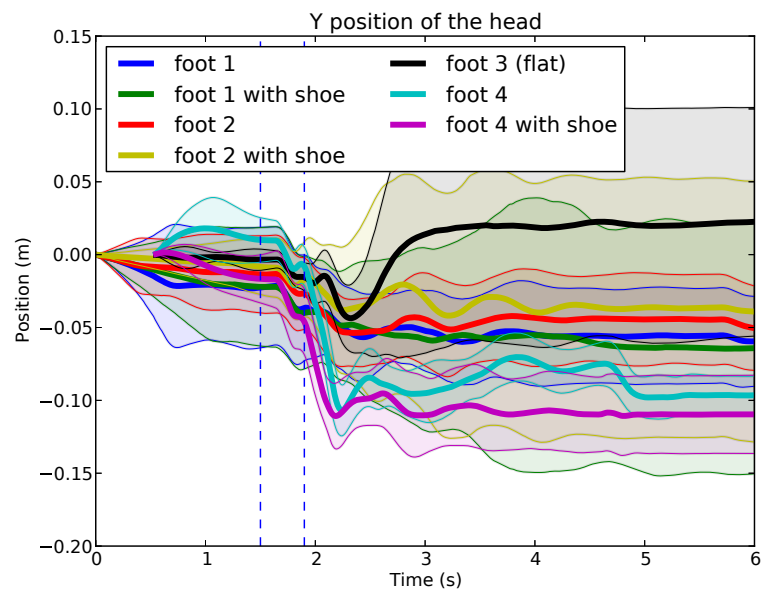

Fig. 5: Evolution of the position of the head in the $y$ axis for each tested foot.

mandatory. Indeed, the observed behaviors of the passive feet were even better than with the flat feet with active rotation. Although a clear interpretation of this phenomenon is still difficult to propose, some clues related to the weight (with one more motor feet are heavier) and ground contact surface (flat foot surface is bigger) have to be investigated.

Moreover, we observed that the shoes added extra friction with the ground without really impairing the stability. Although rarely used in humanoid robotics, these early results encourage us to explore more deeply this possibility.

Finally the most important aspect for us was to actually evaluate the required amount of time needed to conduct such experiment with Poppy. The starting point was the "foot 1 " as it was the in progress work. Thus design modifications of the morphology only concern foot 3 and 4 :

- Foot 3 (flat): Modifying the initial Poppy foot design to permit the integration of two Dynamixel motors and 


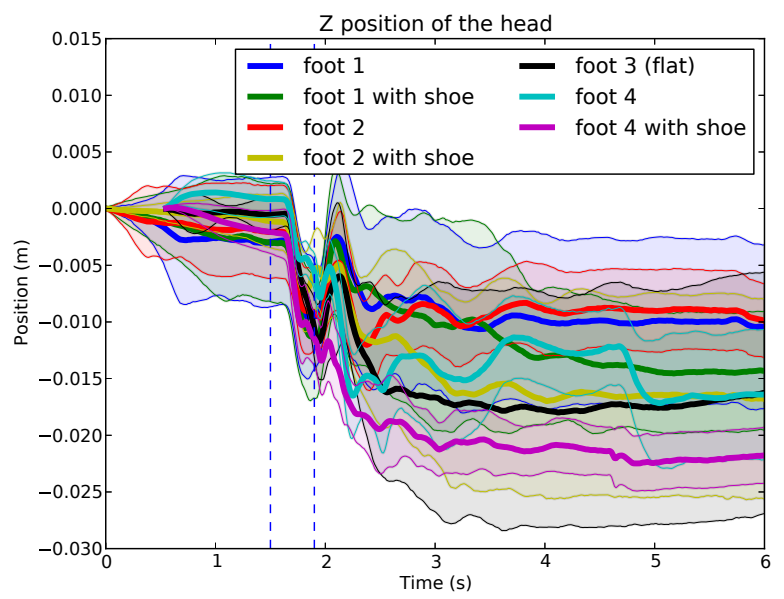

Fig. 6: Evolution of the position of the head in the $z$ axis for each tested foot.

the associated flat feet required $16 \mathrm{~h}$ of CAD design. The printing of the whole needed part ( 2 legs, 2 foot and 2 ankle) took approximately 30 hours on a low cost FDM printer (Makerbot Replicator 2).

- Foot 4: While the difference with the foot 1 concerns only one parameter (i.e. the articulation position), the modification needed to produce the foot 4 based on the foot 1 was done in approximately 2 hours of CAD. The printing of the novel part was achieved in 10 hours.

Then conducting the whole experiment (i.e. design the leg motion, establishment of the experimental setup and data acquisition) was achieved in about one week with two persons. Especially the actual experimentation involving to change seven times the Poppy's feet and acquiring at least 20 trials for each took less than two days.

\section{Reuse of this experiment}

Everything needed to obtain and use Poppy is available on our GitHub project page: www.github.com/poppy_ project. Also to complete the illustration of this Poppy use-case, we diffuse along the present paper:

- the whole setup materials i.e. the code used for the experiment and the 3D files to reproduce/modify each foot,

- the acquired raw data that include for each trial all markers position, head IMU measurement and the complete motors data (proprioceptive position evaluation overtime),

- the code used to extract and plot the presented results.

All these materials are available on the repository associated with this experiment: https://github. com/matthieu-lapeyre/Humanoids2014 and can be freely used e.g. for further investigation with the acquired data, or to reproduce and extend the experiment.

\section{Discussion \& CONCLUSION}

In this paper we presented the motivation and challenges raised by the desire to consider morphology as an experimental variable and at the same time allowing experiments reproduction in the scientific community.

For this purpose we developed Poppy, the first complete 3D printed open-source and open-hardware humanoid robot, designed to allow a free exploration and experimentation of morphological properties in the real world.

The use of 3D printing techniques is a central aspect of the project. This method allows to really consider the morphology as an experimental variable because it is now both cheap and fast to produce several morphological variants. Also, thanks to a modular structure based on particular design, off-the-shell components, Arduino electronics and adapted control API, we can reconfigure Poppy in a couple of minutes and conduct experiment directly in the real world.

A fundamental aspect of our work is to permit the scientific reproducibility of both Poppy and associated experimentation. All our work is distributed under open-source license (Creative commons for hardware and GPLv3 for software) and the chosen techniques rely only on relatively cheap and accessible production methods to ensure the hardware can be easily and quickly reproduced everywhere.

As an illustration of such use, we proposed an experimentation aiming to evaluate and compare the reaction of the robot when it makes a lateral footstep in function of different foot morphologies.

We tested four feet morphologies which were produced by a low cost $3 \mathrm{D}$ printer. We detailed the amount of time required for each step of the experimental process (i.e. design, production, experimentation). Doing such experiment with other platform is usually complicated and costly. With Poppy we managed to explore seven solutions in couple of days. However the acquired data show that the dynamic of the robot reaction is quite complicated and difficult to analyze and would require further investigation. Yet this experimentation was a good use case to illustrate the purpose of Poppy of the robot. To complete the process, we distribute the whole data and material produced for the purpose of this paper in order to make the experimentation fully reproducible and allowing anyone to verify, reuse or create derivative/fork of our initial work.

Thus the philosophy and methodology within the Poppy project permit cumulative and open science. Rather than having to rethink and develop platform or experiment adapted to their challenges, ones can use the openness and modularity of Poppy to adapt it to their needs. They can therefore both spend less time on development and fit in an experimental framework ensuring the reproducibility of their research in the community.

Indeed the future work associated with the Poppy project will be focused on the development of the user community and the continuous improvement of the Poppy platform toward the ease of use, the reproducibility and the modularity. Also as the robot is open source and modular, we are 
expecting to see derivative work using only some module or reconfiguration in non-humanoid form so we are working on web tool allowing to easily track, share and discuss derivative work.

Finally, as it integrates advanced and yet easily accessible techniques in an embodiment that motivates students and the wider public, this platform also meets a growing societal need: education and training in technologies combining computer science, electronics and mechanics, as well as a training tool to the emergent revolutionary 3D printing process. With its openness, its design and its rather lowcost, Poppy provides a unique context for experimentation and learning of these technologies in a Do-It-Yourself (DIY) approach. Therefore the incoming development will also take into account the use case of the Poppy for educational purpose for example in universities and engineering schools.

\section{REFERENCES}

[1] W. G. Walter, "An imitation of Life," 1950.

[2] K. Kaufmann and P. McCorduck, "Machines who think," 1979.

[3] J. Haugeland, Artificial intelligence: The very idea. MIT press, 1989.

[4] R. A. Brooks, "Intelligence without representation," Artificial intelligence, vol. 47, no. 1, pp. 139-159, 1991.

[5] L. Steels and R. A. Brooks, The artificial life route to artificial intelligence: Building embodied, situated agents. Psychology Press, 1995.

[6] R. Pfeifer and C. Scheier, Understanding intelligence. MIT press, 2001.

[7] R. Pfeifer and J. J. C. Bongard, How the Body Shapes the Way We Think: A New View of Intelligence (Bradford Books). The MIT Press, 2006.

[8] P.-Y. Oudeyer, F. Kaplan, V. V. Hafner, and A. Whyte, "The playground experiment: Task-independent development of a curious robot," in Proceedings of the AAAI Spring Symposium on Developmental Robotics. Stanford, California, 2005, pp. 42-47.

[9] T. McGeer, "Passive dynamic walking," The International Journal of Robotics Research, vol. 9, no. 2, pp. 62-82, 1990.

[10] G. Lakoff and R. E. Núñez, Where mathematics comes from: How the embodied mind brings mathematics into being. Basic books, 2000.

[11] C. Paul, "Morphological computation: A basis for the analysis of morphology and control requirements," Robotics and Autonomous Systems, vol. 54, no. 8, pp. 619-630, 2006.

[12] F. Kaplan and P.-Y. Oudeyer, "Le corps comme variable expérimentale," Revue philosophique de la France et de l'étranger, no. 3, pp. 287-298, 2008.

[13] K. Hirai, M. Hirose, Y. Haikawa, and T. Takenaka, "The development of Honda humanoid robot," in Robotics and Automation, 1998. Proceedings. 1998 IEEE International Conference on, vol. 2. IEEE, 1998, pp. 1321-1326.

[14] K. Kaneko, K. Harada, F. Kanehiro, G. Miyamori, and K. Akachi, "Humanoid robot HRP-3," in Intelligent Robots and Systems, 2008. IROS 2008. IEEE/RSJ International Conference on. IEEE, 2008, pp. 2471-2478.

[15] A. Tapus, M. J. Mataric, and B. Scassellati, "Socially assistive robotics," IEEE Robotics and Automation Magazine, vol. 14, no. 1, p. 35, 2007.

[16] M. Asada, K. F. MacDorman, H. Ishiguro, and Y. Kuniyoshi, "Cognitive developmental robotics as a new paradigm for the design of humanoid robots," Robotics and Autonomous Systems, vol. 37, no. 2, pp. 185-193, 2001.

[17] C. Breazeal and B. Scassellati, "Robots that imitate humans," Trends in cognitive sciences, vol. 6, no. 11, pp. 481-487, 2002.

[18] S. S. H. Collins and A. Ruina, "A bipedal walking robot with efficient and human-like gait," in Robotics and Automation, 2005. ICRA 2005. Proceedings of the 2005 IEEE International Conference on. IEEE, 2005, pp. 1983-1988.

[19] A. Ude, C. G. Atkeson, and M. Riley, "Programming full-body movements for humanoid robots by observation," Robotics and autonomous systems, vol. 47, no. 2, pp. 93-108, 2004.
[20] C. G. Atkeson, J. G. Hale, F. E. Pollick, M. Riley, S. Kotosaka, S. Schaul, T. Shibata, G. Tevatia, A. Ude, S. Vijayakumar, and Others, "Using humanoid robots to study human behavior," IEEE Intelligent Systems and their applications, vol. 15, no. 4, pp. 46-56, 2000.

[21] R. A. Brooks, "Achieving artificial intelligence through building robots," 1986.

[22] D. Gouaillier, V. Hugel, P. Blazevic, C. Kilner, J. Monceaux, P. Lafourcade, B. Marnier, J. Serre, and B. Maisonnier, "The nao humanoid: a combination of performance and affordability," CoRR, vol. abs/0807.3223, 2008.

[23] I. Ha, Y. Tamura, H. Asama, J. Han, and D. W. Hong, "Development of open humanoid platform DARwIn-OP," in SICE Annual Conference (SICE), 2011 Proceedings of. IEEE, 2011, pp. 2178-2181.

[24] M. Schwarz, M. Schreiber, S. Schueller, M. Missura, and S. Behnke, "NimbRo-OP Humanoid TeenSize Open Platform," in Proceedings of 7th Workshop on Humanoid Soccer Robots. IEEE-RAS International Conference on Humanoid Robots, 2012.

[25] G. Metta, G. Sandini, D. Vernon, L. Natale, and F. Nori, "The iCub humanoid robot: an open platform for research in embodied cognition," in Proceedings of the 8th workshop on performance metrics for intelligent systems. ACM, 2008, pp. 50-56.

[26] R. Moeckel, S. Pouya, C. Maufroy, F. Peuker, A. Tuleu, S. Bonardi, M. Vespignani, S. Grimmer, P. Aerts, K. D'Aout, H. Hauser, L. Aryananda, R. Pfeifer, K. Stoy, A. Seyfarth, and A. J. Ijspeert, "Locomorph robot system - towards legged modular robots with model-inspired morphology," in Proceedings of the 6th International Symposium on Adaptive Motion in Animals and Machines (AMAM), 2013.

[27] M. Lapeyre, P. Rouanet, and P.-Y. Oudeyer, "The Poppy Humanoid Robot: Leg Design for Biped Locomotion," in IEEE/RSJ International Conference on Intelligent Robots and Systems, Tokyo, Japon, 2013.

[28] Y. Nakanishi, S. Ohta, T. Shirai, Y. Asano, T. Kozuki, Y. Kakehashi, H. Mizoguchi, T. Kurotobi, Y. Motegi, K. Sasabuchi, J. Urata, K. Okada, I. Mizuuchi, M. Inab, and Others, "Design Approach of Biologically-Inspired Musculoskeletal Humanoids," International Journal of Advanced Robotic Systems, vol. 10, no. 216, p. 1, 2013.

[29] M. Wisse, "Three additions to passive dynamic walking: actuation, an upper body, and 3D stability," International Journal of Humanoid Robotics, vol. 2, no. 04, pp. 459-478, 2005.

[30] C. Paul and J. C. Bongard, "The road less travelled: Morphology in the optimization of biped robot locomotion," in Intelligent Robots and Systems, 2001. Proceedings. 2001 IEEE/RSJ International Conference on, vol. 1, IEEE. Ieee, 2001, pp. 226-232.

[31] P.-y. Oudeyer, F. Kaplan, and V. Hafner, "Intrinsic Motivation Systems for Autonomous Mental Development," IEEE Transactions on Evolutionary Computation, vol. 11(2), pp. pp. 265-286, 2007.

[32] C. Moulin-Frier and P.-Y. Oudeyer, "Exploration strategies in developmental robotics: a unified probabilistic framework," in Development and Learning and Epigenetic Robotics (ICDL), 2013 IEEE Third Joint International Conference on. IEEE, 2013, pp. 1-6. 